\title{
Number of complete $N$-ary subtrees on Galton-Watson family trees
}

GEORGE P. YANEV

gyanev@cas.usf.edu

Department of Mathematics, University of South Florida, Tampa, FL 33620, USA

LJUBEN MUTAFCHIEV ljuben@aubg.bg

American University in Bulgaria, 2700 Blagoevgrad, Bulgaria and Institute of Mathematics and Informatics of the Bulgarian Academy of Sciences

\begin{abstract}
We associate with a Bienaymé-Galton-Watson branching process a family tree rooted at the ancestor. For a positive integer $N$, define a complete $N$-ary tree to be the family tree of a deterministic branching process with offspring generating function $s^{N}$. We study the random variables $V_{N, n}$ and $V_{N}$ counting the number of disjoint complete $N$-ary subtrees, rooted at the ancestor, and having height $n$ and $\infty$, respectively. Dekking (1991) and Pakes and Dekking (1991) find recursive relations for $P\left(V_{N, n}>0\right)$ and $P\left(V_{N}>0\right)$ involving the offspring probability generation function (pgf) and its derivatives. We extend their results determining the probability distributions of $V_{N, n}$ and $V_{N}$. It turns out that they can be expressed in terms of the offspring pgf, its derivatives, and the above probabilities. We show how the general results simplify in case of fractional linear, geometric, Poisson, and one-or-many offspring laws.
\end{abstract}

Keywords: branching process - family tree - binary tree - $N$-ary tree

AMS 2000 Subject Classification: Primary: 60J80. Secondary: 05 C05.

\section{Introduction and main results}

Consider the family tree associated with a Bienaymé-Galton-Watson process with the following simple reproduction rules. At generation zero, the process starts with single ancestor called root of the tree. Then each individual in the population has, independently of the others, a random number $X$ of children distributed according to the offspring distribution with probability generating function (pgf)

$$
f(s)=\sum_{k=0}^{\infty} p_{k} s^{k},
$$


satisfying $f(1)=1$. Further on we adopt the well-known construction of a family tree generated by a simple branching process where the individuals are the nodes and the parent-child relations define the arcs of the tree in the following manner, see e.g. Harris (1963), Ch.7. Let the $i$ th child of the ancestor be $(i)$ and in general $\left(i_{1} i_{2} \ldots i_{k-1} i_{k}\right)$ denotes the $i_{k}$ th child of $\left(i_{1} i_{2} \ldots i_{k-1}\right)$. Then, a directed arc is assumed to emanate from $\left(i_{1} i_{2} \ldots i_{k-1}\right)$ to $\left(i_{1} i_{2} \ldots i_{k-1} i_{k}\right)$. Since, in our case, the children appear simultaneously, we suppose that the ordering is performed by a chance device independently of the evolution in the process. This scheme produces family trees (also called rooted ordered trees) in which the nodes of height (also known as depth) $n(n \geq 0)$ have labels $\left(i_{1} i_{2} \ldots i_{n}\right)$, with the ancestor (root) having height 0 . The height of a subtree equals the maximum height of its nodes.

For fixed integer $N \geq 1$, define a complete infinite $N$-ary tree to be the family tree of a deterministic branching process with offspring pgf $f(s)=s^{N}$. Further on we will consider rooted subtrees of a family tree. Two such subtrees are called disjoint if they do not have a common node different from the root. These kinds of trees appear, for example, in some computer algorithms; for more details see Knuth (1997).

Let $\left\{Z_{n}: n \geq 1 ; Z_{0}=1\right\}$ denote the generation size process, and let $T_{N}-1$ be the height of a complete $N$-ary subtree rooted in the ancestor; $T_{N}=0$ if $Z_{1}<N$. Notice that $T_{1}$ is the extinction time of $\left\{Z_{n}\right\}$. The study of the probability $\tau_{N}=\lim _{n \rightarrow \infty} P\left(T_{N}>n\right)$ that a BienayméGalton-Watson tree contains an infinite complete $N$-ary subtree was initiated by Dekking (1991) who considered complete binary $(N=2)$ subtrees. The general $(N \geq 2)$ case was subsequently investigated in detail by Pakes and Dekking (1991). In particular, they encountered the following phenomenon: if $N \geq 2$, then there is a critical value $m_{N}^{c}$ for the offspring mean $m=f^{\prime}(1)$ such that $\tau_{N}=0$ if $m<m_{N}^{c}$ and $\tau_{N}>0$ if $m \geq m_{N}^{c}$. This is qualitatively different from what happens for $N=1$ where the probability for non-extinction $\tau_{1}=0$ if $m=m_{1}^{c}=1$, except for the trivial case where $f(s)=s$. Our work is motivated by the results of Pakes and Dekking (1991).

We introduce the random variable $V_{N}$ to be the number of disjoint complete $N$-ary subtrees with infinite height, rooted at the ancestor of a Bienaymé-Galton-Watson family tree. Clearly $\tau_{N}=P\left(V_{N}>0\right)$. As usual, we assume for the offspring distribution $\left\{p_{k}\right\}_{k=0}^{\infty}$ that $p_{k}<1$ for all $k$ and $p_{k}>0$ for some $k>N$. Let $\mathcal{N}$ be the set of all positive integers and denote for $x, y \geq 0$ and any $j=0,1, \ldots$

$$
G_{N}(x, y ; j)=\sum_{k=j N}^{j N+N-1} \frac{x^{k}}{k !} f^{(k)}(y) .
$$

Pakes and Dekking (1991) showed that $P\left(V_{N}=0\right)=1-\tau_{N}$, where $1-\tau_{N}$ is the smallest 
solution in $[0,1]$ of the equation

$$
x=G_{N}(1-x, x ; 0) .
$$

Our goal is to study the distribution of $V_{N}$. As the following result shows, the probability mass function (pmf) of $V_{N}$ can be obtained using the Taylor expansion of $f(1)$ about the point $1-\tau_{N}$.

Theorem 1 If $N \in \mathcal{N}$ then for any $j=0,1, \ldots$

$$
P\left(V_{N}=j\right)=G_{N}\left(\tau_{N}, 1-\tau_{N} ; j\right)
$$

and $P\left(V_{N}=0\right)=1-\tau_{N}$ is the smallest solution in $[0,1]$ of (1).

Remark (i) If $N=1$, then obviously $P\left(V_{1}=0\right)=1-\tau_{1}=q$ is the extinction probability of the Galton-Watson process. Now, (2) becomes

$$
P\left(V_{1}=j\right)=\frac{(1-q)^{j}}{j !} f^{(j)}(q), \quad j=0,1, \ldots,
$$

which in turn implies that $E\left(s^{V_{1}}\right)=f(q+(1-q) s)$. This identity follows directly observing that the number of distinct infinite unary trees is equal to the number of first generation nodes having infinite line of descent. ${ }^{1}$

(ii) Also note that a sufficient condition for $P\left(V_{N}=0\right)<1$ is given in Pakes and Dekking (1991), Theorem 3. In particular, they show that $P\left(V_{N}=0\right)<1(N \geq 2)$ if

$$
2 N \sum_{j \geq N} \frac{p_{j}}{j+1} \leq\left(1-\sum_{j=0}^{N-1} p_{j}\right)^{2} .
$$

The number of complete $N$-ary subtrees is a measure for the rate of growth (or fertility) of the branching process. In fact, as was pointed out in Dekking (1991), if $P\left(V_{2}>0\right)>0$ then we can say that the branching process grows faster than binary splitting. In the study of the tree structure of branching processes, an important role is played by the process' total progeny. Denote by $\nu_{n}$ the number of individuals who existed in the first $n+1$ generations, i.e., $\nu_{n}=1+Z_{1}+\ldots+Z_{n}, n=1,2, \ldots$ Obviously, $\nu_{n}$ equals the total number of nodes having height less than or equal to $n$. Let us also define the random variable $V_{N, n}$ to be the number of disjoint complete $N$-ary subtrees of height at least $n$ rooted at the ancestor of a Bienaymé-Galton-Watson family tree. Let

$$
\psi_{N, n}(s)=E\left(s^{\nu_{n}} ; V_{N, n}>0\right) \quad \text { and } \quad \phi_{N, n}(s)=E\left(s^{\nu_{n}} ; V_{N, n}=0\right) .
$$

The following result presents a recursive relation for the joint distribution of $V_{N, n}$ and $\nu_{n}$.

\footnotetext{
${ }^{1}$ The authors are indebted to the referee who pointed out this argument. It implies immediately the result of Theorem 1 for unary trees.
} 
Theorem 2 If $N \in \mathcal{N}$ then for $|s| \leq 1$ and any $j=0,1, \ldots$

$$
E\left(s^{\nu_{n+1}} ; V_{N, n+1}=j\right)=s G_{N}\left(\psi_{N, n}(s), \phi_{N, n}(s) ; j\right) .
$$

Notice that, if $N=1$ and $j=0$, then the above recurrence reduces to the well-known $E\left(s^{\nu_{n+1}} ; Z_{n+1}=0\right)=s f\left(E\left(s^{\nu_{n}} ; Z_{n}=0\right)\right)$, see e.g. Kolchin (1986), p. 120.

Applications of complete $N$-ary trees can be found in the analysis of algorithms, see Knuth (1997). Problems of this nature appear also in percolation theory. For instance, Pakes and Dekking (1991) point out a relationship between the model of $N$-ary complete and infinite subtrees and a construction employed by Chayes et al. (1988) in their study of Mandelbrot's percolation processes. The existence of $N$-ary subtrees is also used by Pemantle (1988) in introducing the concept of a $N$-infinite branching process. Let us also mention potential connections with problems of percolation of binary words on the nodes of locally finite graphs with countably infinite node-sets, see Benjamini and Kesten (1995).

We organize our paper as follows. In Section 2 we prove the main results. Sections 3-5 contain some illustrations. In Section 3 we consider the family tree generated by the fractional linear $f(s)$ as well as the special case of geometric offspring. In the latter case, $V_{N}$ itself follows a geometric distribution. It turns out that in the Poisson offspring case, given in Section 4, the pmf of $V_{N}$ can be expressed in terms of certain Poisson probabilities. Note that the critical values $m_{N}^{c}(N \geq 2)$ in the Poisson case are less than those in the geometric one. Finally, in Section 5 we consider the one-or-many (i.e., concentrated on two points only) offspring distribution. In this case $V_{N}$ has a pmf given in terms of binomial probabilities.

\section{Proofs of the Theorems}

Proof of Theorem 1 Let us consider $P\left(V_{N}=j\right)$ where $j=1,2, \ldots$ Recall that the random variable $V_{N, n}$ equals the number of disjoint complete $N$-ary subtrees of height $n$ rooted at the ancestor of a Bienaymé-Galton-Watson family tree. First, we will find the pmf of $V_{N, n+1}$ using the total probability formula. Indeed, to have $j$ disjoint complete $N$-ary subtrees rooted at the ancestor node there must be $j N+k(k \geq 0)$ nodes in the first generation. Each of these nodes can be considered as an ancestor of a family tree rooted at the first generation. Consider the event $A_{N}(l)=\left\{j N+l\right.$ of the $Z_{1}$ first generation nodes are ancestors of at least one complete $N$-ary tree of height $n\}$, where $l=0,1, \ldots, \min \{k, N-1\}$. If $Z_{1}=j N+k$ then for fixed $l$ the event $A_{N}(l)$ has conditional probability

$$
P\left(A_{N}(l) \mid Z_{1}=j N+k\right)=\left(\begin{array}{c}
j N+k \\
j N+l
\end{array}\right)\left(\tau_{N, n}\right)^{j N+l}\left(1-\tau_{N, n}\right)^{k-l} \quad(0 \leq l \leq \min \{k, N-1\}),
$$


where $\tau_{N, n}=1-P\left(V_{N, n}=0\right)$ and by convention let $\tau_{N, 0}=1$. We have

$$
P\left(\bigcup_{l=0}^{\min \{k, N-1\}} A_{N}(l) \mid Z_{1}=j N+k\right)=\sum_{l=0}^{\min \{k, N-1\}}\left(\begin{array}{c}
j N+k \\
j N+l
\end{array}\right)\left(\tau_{N, n}\right)^{j N+l}\left(1-\tau_{N, n}\right)^{k-l} .
$$

Applying the total probability formula and changing the order of summation, we obtain

$$
\begin{aligned}
P\left(V_{N, n+1}=j\right) & =\sum_{k=0}^{\infty} P\left(Z_{1}=j N+k\right) P\left(\bigcup_{l=0}^{\min \{k, N-1\}} A_{N}(l) \mid Z_{1}=j N+k\right) \\
& =\sum_{k=0}^{\infty} p_{j N+k}\left\{\sum_{l=0}^{\min \{k, N-1\}}\left(\begin{array}{c}
j N+k \\
j N+l
\end{array}\right)\left(\tau_{N, n}\right)^{j N+l}\left(1-\tau_{N, n}\right)^{k-l}\right\} \\
& =\sum_{l=0}^{N-1} \frac{\tau_{N, n}^{j N+l}}{(j N+l) !} \sum_{k=l}^{\infty} p_{j N+k}(j N+k)(j N+k-1) \ldots(k-l+1)\left(1-\tau_{N, n}\right)^{k-l} \\
& =\sum_{l=0}^{N-1} \frac{\tau_{N, n}^{j N+l}}{(j N+l) !} f^{(j N+l)}\left(1-\tau_{N, n}\right) \\
& =G_{N}\left(\tau_{N, n}, 1-\tau_{N, n} ; j\right) .
\end{aligned}
$$

By definition $\tau_{N, 0}=1$ and $\tau_{N, n} \downarrow \tau_{N}$ as $n \uparrow \infty$. Letting $n \rightarrow \infty$, we obtain for $j \geq 1$

$$
P\left(V_{N}=j\right)=\lim _{n \rightarrow \infty} P\left(V_{N, n+1}=j\right)=G_{N}\left(\tau_{N}, 1-\tau_{N} ; j\right) .
$$

Let us now consider the case $j=0$. The above recurrence is true for $n=0$, i.e., $P\left(V_{N, 1}=0\right)=$ $G_{N}(1,0 ; 0)=\sum_{k=0}^{N-1} p_{k}$. For $n \geq 1$, using the total probability formula and an argument similar to that for the case $j \geq 1$, we obtain

$$
\begin{aligned}
P\left(V_{N, n+1}=0\right) & =\sum_{l=0}^{N-1} \sum_{k=l}^{\infty} p_{k}\left(\begin{array}{l}
k \\
l
\end{array}\right)\left(\tau_{N, n}\right)^{l}\left(1-\tau_{N, n}\right)^{k-l} \\
& =\sum_{l=0}^{N-1} \frac{\left(\tau_{N, n}\right)^{l}}{l !} f^{(l)}\left(1-\tau_{N, n}\right) \\
& =G_{N}\left(\tau_{N, n}, 1-\tau_{N, n} ; 0\right) .
\end{aligned}
$$

Computing the derivative of $G_{N}(x, 1-x ; 0)$, we get a telescoping sum which after cancelations becomes $d G_{N}(x, 1-x ; 0) / d x=(1-x)^{N-1} f^{(N)}(x) /(N-1) ! \geq 0$ for $0 \leq x \leq 1$. Thus, $G_{N}(x, 1-$ $x ; 0)$ is non-decreasing in $[0,1]$, and therefore

$$
1-\tau_{N}=\lim _{n \rightarrow \infty}\left(1-\tau_{N, n+1}\right)=\lim _{n \rightarrow \infty} P\left(V_{N, n+1}=0\right)=G_{N}\left(\tau_{N}, 1-\tau_{N} ; 0\right)
$$

is the smallest root in $[0,1]$ of the equation $x=G_{N}(1-x, x ; 0)$. The proof is complete.

Clearly (2) implies that $\sum_{j=0}^{\infty} P\left(V_{N}=j\right)=\sum_{k=0}^{\infty} \tau_{N}^{k} f^{(k)}\left(1-\tau_{N}\right) / k !=f(1)=1$.

Proof of Theorem 2 Let us introduce the notation

$$
\tau_{N, n}(t)=P\left(V_{N, n}>0, \nu_{n}=t\right), \quad \gamma_{N, n}(t)=P\left(V_{N, n}=0, \nu_{n}=t\right)=P\left(\nu_{n}=t\right)-\tau_{N, n}(t),
$$


where $N, n$, and $t$ are positive integers. Proceeding as in the proof of Theorem 1 , we consider the event

$$
A_{N}(l, t)=A_{N}(l) \bigcap\left\{\nu_{n+1}=t\right\}
$$

where $A_{N}(l)$ is defined in the proof of Theorem 1. For fixed $t$ and $l(0 \leq l \leq \min (k, N-1))$, using the fact that all trees rooted in the first generation grow independently, we compute the conditional probability of $A_{N}(l, t)$ given $Z_{1}=j N+k$ to be

$$
P\left(A_{N}(l, t) \mid Z_{1}=j N+k\right)=\left(\begin{array}{c}
j N+k \\
j N+l
\end{array}\right) \sum^{\prime} \prod_{u=1}^{j N+l} \tau_{N, n}\left(n_{u}\right) \prod_{v=j N+l+1}^{j N+k} \gamma_{N, n}\left(n_{v}\right),
$$

where the summation in $\sum^{\prime}$ is over all nonnegative integers $\left\{n_{i}\right\}_{i=1}^{j N+k}$ such that $\sum_{i=1}^{j N+k} n_{i}=t-1$. Then, the total probability formula implies that

$$
\begin{aligned}
P\left(V_{N, n+1}=j, \nu_{n+1}=t\right) & =\sum_{k=0}^{\infty} P\left(Z_{1}=j N+k\right) \sum_{l=0}^{\min (k, N-1)} P\left(A_{N}(l, t) \mid Z_{1}=j N+k\right) \\
& =\sum_{l=0}^{N-1} \sum_{k=l}^{\infty} p_{j N+k}\left(\begin{array}{c}
j N+k \\
j N+l
\end{array}\right) \sum^{\prime} \prod_{u=1}^{j N+l} \tau_{N, n}\left(n_{u}\right) \prod_{v=j N+l+1}^{j N+k} \gamma_{N, n}\left(n_{v}\right) .
\end{aligned}
$$

Multiplying both sides of this equality by $s^{t}$ and summing over $t$, we get

$$
\begin{aligned}
E\left(s^{\nu_{n+1}} ; V_{N, n+1}=j\right)= & s \sum_{l=0}^{N-1} \frac{1}{(j N+l) !} \sum_{k=l}^{\infty} p_{j N+k}(j N+k)(j N+k-1) \ldots(k-l+1) \\
& \times \sum_{t=1}^{\infty} \sum^{\prime} \prod_{u=1}^{j N+l} \tau_{N, n}\left(n_{u}\right) \prod_{v=j N+l+1}^{j N+k} \gamma_{N, n}\left(n_{v}\right) s^{t-1} .
\end{aligned}
$$

Observe that the coefficient of $s^{t-1}$ in the series

$$
\sum_{t=1}^{\infty} \sum^{\prime} \prod_{u=1}^{j N+l} \tau_{N, n}\left(n_{u}\right) \prod_{v=j N+l+1}^{j N+k} \gamma_{N, n}\left(n_{v}\right) s^{t-1}
$$

can be written as

$$
\sum_{h=0}^{t-1} \sum_{n_{1}+\ldots+n_{j N+l}=h} \prod_{u=1}^{j N+l} \tau_{N, n}\left(n_{u}\right) \sum_{n_{j N+l+1}+\ldots+n_{j N+k}=t-1-h} \prod_{v=j N+l+1}^{j N+k} \gamma_{N, n}\left(n_{v}\right) .
$$

The rule of multiplying power series implies that this coefficient equals the coefficient of $s^{t-1}$ in the power series expansion of

$$
\left[\sum_{i=1}^{\infty} \tau_{N, n}(i) s^{i}\right]^{j N+l}\left[\sum_{i=1}^{\infty} \gamma_{N, n}(i) s^{i}\right]^{k-l}=\left[\psi_{N, n}(s)\right]^{j N+l}\left[\phi_{N, n}(s)\right]^{k-l},
$$

where $\psi_{N, n}(s)$ and $\phi_{N, n}(s)$ are defined in (3). Therefore,

$$
\begin{aligned}
E\left(s^{\nu_{n+1}} ; V_{N, n+1}=j\right) & =s \sum_{l=0}^{N-1} \frac{\left[\psi_{N, n}(s)\right]^{j N+l}}{(j N+l) !} \sum_{k=l}^{\infty} p_{j N+k}(j N+k)(j N+k-1) \ldots(k-l+1)\left[\phi_{N, n}(s)\right]^{k-l} \\
& =s \sum_{l=0}^{N-1} \frac{\left[\psi_{N, n}(s)\right]^{j N+l}}{(j N+l) !} f^{(j N+l)}\left(\phi_{N, n}(s)\right)
\end{aligned}
$$


which coincides with the right-hand side of (4). This completes the proof.

\section{$3 \quad$ Fractional linear offspring}

Let $f(s)$ be a fractional linear pgf given by

$$
f(s)=1-\frac{b}{1-p}+\frac{b s}{1-p s}
$$

and the parameter space $\{(p, b): 0<p<1,0<b \leq 1-p\}$. Then the offspring distribution is given by the geometric series $p_{k}=b p^{k-1}, k=1,2, \ldots ; p_{0}=1-\sum_{k=1}^{\infty} p_{k}$ and the offspring mean is $m=b /(1-p)^{2}$. In the particular case $b=p(1-p)$ we have $p_{k}=(1-p) p^{k}, k \geq 0$ which is the standard geometric distribution with pgf $f(s)=(1-p) /(1-p s)$. It can be verified, see Pakes and Dekking (1991), p. 361 if $N \geq 2$ and Harris (1963), p. 9 if $N=1$, that for $N \in \mathcal{N}$

$$
1-p\left(1-\tau_{N}\right)=[b /(1-p)]^{1 / N}\left[p \tau_{N}\right]^{1-1 / N} .
$$

Proposition 1 If the offspring distribution has the fractional linear pgf (6), then $V_{N}$ follows a zero-modified geometric (i.e., fractional linear) distribution given by

$$
P\left(V_{N}=j\right)=\frac{b}{p(1-p)}\left(1-\theta_{N}\right) \theta_{N}^{j} \quad(j \geq 1), \quad P\left(V_{N}=0\right)=1-\frac{b}{p(1-p)} \theta_{N}
$$

and

$$
E V_{N}=\frac{b}{p(1-p)} \frac{\theta_{N}}{1-\theta_{N}}
$$

where

$$
\theta_{N}=\left(\frac{p \tau_{N}}{1-p\left(1-\tau_{N}\right)}\right)^{N}
$$

and $\tau_{N}$ is the largest solution in $[0,1]$ of (7).

Proof Since $f^{(i)}(s)=i ! b p^{i-1} /(1-p s)^{i+1} \quad(i \geq 1)$, we have from (2) for $j \geq 1$

$$
\begin{aligned}
P\left(V_{N}=j\right) & =\sum_{k=0}^{N-1} \frac{\tau_{N}^{j N+k}}{(j N+k) !} \frac{b(j N+k) ! p^{j N+k-1}}{\left(1-p\left(1-\tau_{N}\right)\right)^{j N+k+1}} \\
& =\frac{b p^{j-1} \tau_{N}^{j N}}{\left(1-p\left(1-\tau_{N}\right)\right)^{j N+1}} \sum_{k=0}^{N-1} \frac{\left(p \tau_{N}\right)^{k}}{\left(1-p\left(1-\tau_{N}\right)\right)^{k}} .
\end{aligned}
$$

Now, setting $\left(\theta_{N}\right)^{1 / N}=p \tau_{N} /\left(1-p\left(1-\tau_{N}\right)\right)$ one can obtain the first formula in (8) , which in turn leads to (8) and (9).

Corollary If the offspring distribution is geometric, i.e., $p_{k}=(1-p) p^{k}, k \geq 0$, then $V_{N}$ is geometric as well, $P\left(V_{N}=j\right)=\left(1-\tau_{N}\right) \tau_{N}^{j}(j \geq 0)$ and $E V_{N}=\tau_{N}\left(1-\tau_{N}\right)^{-1}$, where $\tau_{N}$ is the largest solution in $[0,1]$ of $\left(\tau_{N}+1 / m\right)^{N}=\tau_{N}^{N-1} \quad(N \geq 1)$. 
Proof In the case of geometric offspring (6) holds with $b=p(1-p)$ and $m=p /(1-p)$. The equation for $\tau_{N}$ follows by inspection from (17). It is also given in Pakes and Dekking (1991), p.361 if $N \geq 2$. Simple algebraic manipulations show that this equation simplifies to $\theta_{N}=\tau_{N}$. Now, the rest of the statement follows from (8) and (9).

Remark For geometric offspring with mean $m>1$ we have $P\left(V_{1}=j\right)=(1 / m)(1-1 / m)^{j}$ and $E V_{1}=m-1$. In particular, $P\left(V_{1}=0\right)=1 / m$ which equals the probability of extinction, see Harris (1963), p. 9.

Table 1 lists the probabilities $P\left(V_{N}=j\right), j=0,1,2, \ldots 9$ as well as $E V_{N}$ for $1 \leq N \leq 5$. The critical mean values (see Section 1) are as follows: $m_{1}^{c}=1, m_{2}^{c}=4, m_{3}^{c}=6.75, m_{4}^{c}=9.481$, $m_{5}^{c}=12.207$. The expected values in the last column provide a measure of how many $N$-ary subtrees $(1 \leq N \leq 5)$ are supported by the geometric family tree with offspring mean fixed to be $m=13$. See also Table 2 below for a comparison with the Poisson offspring case.

\begin{tabular}{|c|c|c|c|c|c|c|c|c|c|c|c|c|}
\hline$V_{N}=$ & 0 & 1 & 2 & 3 & 4 & 5 & 6 & 7 & 8 & 9 & $\geq 10$ & $E\left(V_{N}\right)$ \\
\hline$N=1$ & 0.08 & 0.07 & 0.07 & 0.06 & 0.06 & 0.05 & 0.05 & 0.04 & 0.04 & 0.04 & 0.44 & 12 \\
\hline$N=2$ & 0.16 & 0.14 & 0.11 & 0.10 & 0.08 & 0.07 & 0.06 & 0.05 & 0.04 & 0.03 & 0.16 & 5.22 \\
\hline$N=3$ & 0.26 & 0.19 & 0.14 & 0.11 & 0.08 & 0.06 & 0.04 & 0.03 & 0.02 & 0.02 & 0.05 & 2.91 \\
\hline$N=4$ & 0.37 & 0.23 & 0.15 & 0.09 & 0.06 & 0.04 & 0.02 & 0.01 & 0.01 & 0.01 & 0.01 & 1.71 \\
\hline$N=5$ & 0.53 & 0.25 & 0.12 & 0.05 & 0.03 & 0.01 & 0.01 & 0 & 0 & 0 & 0 & 0.87 \\
\hline
\end{tabular}

Table 1: Probability distribution of $V_{N}$ assuming geometric offspring with $m=13$.

\section{Poisson offspring}

Consider the case of Poisson offspring distribution with pgf given by

$$
f(s)=e^{m(s-1)} \quad(m>0) .
$$

Then, the probability $\tau_{N}$ is the largest solution of

$$
(1-s) e^{m s}=\sum_{j=0}^{N-1}(m s)^{j} / j !
$$

(see Pakes and Dekking (1991), p. 364). Since $f^{(i)}(s)=m^{i} e^{m(s-1)}(i \geq 0)$, formula (2) becomes

$$
P\left(V_{N}=j\right)=e^{-m \tau_{N}} \sum_{k=0}^{N-1} \frac{\left(m \tau_{N}\right)^{j N+k}}{(j N+k) !}, j \geq 0 .
$$


Therefore, we have the following

Proposition 2 If the offspring distribution has the Poisson pgf (10), then

$$
P\left(V_{N}=j\right)=P\left(j N \leq Y_{N} \leq j N+N-1\right),
$$

where $Y_{N}$ has the Poisson pmf

$$
P\left(Y_{N}=k\right)=\left(m \tau_{N}\right)^{k} e^{-m \tau_{N}} / k ! \quad k=0,1,2, \ldots
$$

and $\tau_{N}$ is the largest solution in $[0,1]$ of equation (11).

Notice that $V_{1}$ has a Poisson distribution with parameter $m \tau_{1}$. To calculate the critical value $m_{N}^{c}$ that yields a non-zero solution $\tau_{N}^{c}$ in $[0,1]$ of equation (111) we first notice that the product $y=m_{N}^{c} \tau_{N}^{c}$ satisfies the equations

$$
y^{N} /(N-1) !+\sum_{j=0}^{N-1} y^{j} / j !=e^{y} ;
$$

see Pakes and Dekking (1991), p. 365. Following their way of calculation, one can find $m_{N}^{c}$ and $\tau_{N}^{c}$ by substituting the solution of (12) into

$$
m y^{N-1} /(N-1) !=e^{y}
$$

In case of binary trees, one can also use the Cayley's tree function $y(z)=\sum_{k=1}^{\infty} k^{k-1} z^{k} / k$ ! (see e.g. Odlyzko (1995), Section 6.2) evaluated at $z=1 / m_{N}^{c}$ for the solution of (12). Inserting it into (12), we obtain $m_{2}^{c}=3.3509$ and $\tau_{2}^{c}=0.5352$.

Our final remark concerns the case $m \rightarrow \infty$. It is easily seen that Proposition 2 and the normal approximation of the Poisson distribution imply a local limit theorem for $V_{N}$. Moreover, Pakes and Dekking (1991) showed that in this case $\tau_{N} \rightarrow 1$. This enables one to centralize and scale the limiting variable $V_{N}$ in terms of the single parameter $m$ only.

Table 2 gives the probabilities $P\left(V_{N}=j\right), j=0,1,2, \ldots 9$ as well as $E V_{N}$ for $2 \leq N \leq 5$. The critical mean values are as follows: $m_{2}^{c}=3.3509, m_{3}^{c}=5.1494, m_{4}^{c}=6.7993, m_{5}^{c}=8.3653$.

\begin{tabular}{|c|c|c|c|c|c|c|c|c|c|c|c|c|}
\hline$V_{N}=$ & 0 & 1 & 2 & 3 & 4 & 5 & 6 & 7 & 8 & 9 & $\geq 10$ & $E\left(V_{N}\right)$ \\
\hline$N=2$ & 0 & 0 & 0.01 & 0.04 & 0.11 & 0.19 & 0.22 & 0.19 & 0.13 & 0.07 & 0.04 & 6.25 \\
\hline$N=3$ & 0 & 0.01 & 0.09 & 0.25 & 0.32 & 0.22 & 0.08 & 0.02 & 0 & 0 & 0.01 & 4.00 \\
\hline$N=4$ & 0 & 0.05 & 0.30 & 0.41 & 0.19 & 0.04 & 0 & 0 & 0 & 0 & 0.01 & 2.87 \\
\hline$N=5$ & 0 & 0.17 & 0.51 & 0.28 & 0.04 & 0 & 0 & 0 & 0 & 0 & 0 & 2.19 \\
\hline
\end{tabular}

Table 2: Probability distribution of $V_{N}$ assuming Poisson offspring with $m=13$. 


\section{One-or-many offspring}

In this section we consider a two-parameter family of 1-or-r offspring distributions defined for some $p \in(0,1)$ by $p_{1}=1-p$ and $p_{r}=p$, where $r>N>1$. Its pgf is $f(s)=(1-p) s+p s^{r}$ and thus $f^{\prime}(s)=1-p+p r s^{r-1}$ and $f^{(k)}(s)=p r(r-1) \ldots(r-k+1) s^{r-k}(2 \leq k \leq r)$. The probability $\tau_{N}$ is the largest solution in $[0,1]$ of

$$
s=p \sum_{k=N}^{r}\left(\begin{array}{l}
r \\
k
\end{array}\right) s^{k}(1-s)^{r-k}
$$

(see again Pakes and Dekking (1991), p.366). Applying (2) it is not difficult to obtain

$$
P\left(V_{N}=0\right)=1-p+p \sum_{k=0}^{N-1}\left(\begin{array}{l}
r \\
k
\end{array}\right) \tau_{N}^{k}\left(1-\tau_{N}\right)^{r-k}
$$

and for $j=1,2, \ldots$ and $r \geq j N$

$$
P\left(V_{N}=j\right)=p \sum_{k=j N}^{j N+U}\left(\begin{array}{l}
r \\
k
\end{array}\right) \tau_{N}^{k}\left(1-\tau_{N}\right)^{r-k},
$$

where $U=\min \{N-1, r-j N\}$. Let $B_{r}\left(\tau_{N}\right)$ denote a binomial $\left(r, \tau_{N}\right)$ random variable.

Proposition 3 If the offspring pgf is $f(s)=(1-p) s+p s^{r}(1 \leq N<r)$ and $\tau_{N}$ is the largest solution in $[0,1]$ of (14), then $P\left(V_{N}=0\right)=1-p+p P\left(B_{r}\left(\tau_{N}\right) \leq N-1\right)$ and for $j=1,2, \ldots$

$$
P\left(V_{N}=j\right)=p P\left(j N \leq B_{r}\left(\tau_{N}\right) \leq j N+U\right) \quad \text { if } j N \leq r
$$

where $U=\min \{N-1, r-j N\}$ and $P\left(V_{N}=j\right)=0$ if $j N>r$. The expected value of $V_{N}$ is

$$
E V_{N}=p \sum_{j=1}^{[r / N]} j P\left(j N \leq B_{r}\left(\tau_{N}\right) \leq j N+U\right),
$$

where $[x]$ is the integer part of $x$.

In particular, if $r=N+1$ or $r=N+2$ and $N>2$, then (15) implies that $V_{N}$ takes on values 0 or 1 ; if $N=2$ and $r=4$, then $V_{N}$ takes on values 0,1 , or 2 . Table 3 provides some numerical illustrations. Note that the offspring mean $m=13.09$ enables comparisons with Tables 1 and 2 .

It is interesting to point out the following relationship between the 1-or-r and Poisson offspring cases. There exists (see Pakes and Dekking (1991)) a critical value $p_{N}^{c}$ such that for $p=p_{N}^{c}$ equation (14) has a single solution $\tau_{N}^{c}$ in $(0,1)$. Suppose that $\lim _{r \rightarrow \infty}\left(r \tau_{N}^{c}\right) \rightarrow y$, where $y$ satisfies (13) and (12). Then, applying Theorem 7, Pakes and Dekking (1991), one can obtain that $V_{N}(r)$ converges in distribution to $V_{N}(y)$, where $V_{N}(r)$ and $V_{N}(y)$ are copies of $V_{N}$ assuming one-or-many and Poisson offspring with mean $m_{N}^{c}$, respectively. 


\begin{tabular}{|c|c|c|c|c|c|c|c|c|c|}
\hline$V_{N}=$ & 0 & 1 & 2 & 3 & 4 & 5 & 6 & 7 & $E\left(V_{N}\right)$ \\
\hline$N=2$ & 0.07 & 0 & 0 & 0 & 0 & 0.06 & 0.53 & 0.34 & 5.86 \\
\hline$N=3$ & 0.07 & 0 & 0 & 0.06 & 0.87 & 0 & 0 & 0 & 5.05 \\
\hline$N=4$ & 0.07 & 0 & 0.06 & 0.87 & 0 & 0 & 0 & 0 & 2.73 \\
\hline$N=5$ & 0.07 & 0 & 0.93 & 0 & 0 & 0 & 0 & 0 & 1.86 \\
\hline
\end{tabular}

Table 3: Probability distribution of $V_{N}$ assuming 1-or-14 offspring with $p=0.93(m=13.09)$.

\section{Acknowledgments}

We thank the referee for his valuable comments and suggestions and especially for his help to eliminate some defects in Proposition 1. This work was done during L. Mutafchiev's visit at the Mathematics Department of the University of South Florida in 2004-05 academic year. He thanks for the hospitality and support. G. Yanev is partially supported by NFSI-Bulgaria, MM-1101/2001.

\section{References}

I. Benjamini and H. Kesten, "Percolation of arbitrary words in $\{0,1\}^{N}$," Ann. Probab. vol. 23, 1024-1060, 1995.

J.L. Chayes, L. Chayes and R. Durret, "Connectivity properties of Mandelbrot's percolation process," Prob. Theor. Rel. Fields vol. 77, 307-324, 1988.

F.M. Dekking, "Branching processes that grow faster than binary splitting,", Amer. Math. Monthly vol. 98, 728-731, 1991.

T.E. Harris, The Theory of Branching Processes, Springer, Berlin, 1963.

D.E. Knuth, The Art of Computer Programming, vol. 1: Fundamental Algorithms, 3rd ed., Addison-Wesley, Reading, Mass, 1997.

V.F. Kolchin, Random Mappings, Optimization Software, Inc., New York, 1986.

A.M. Odlyzko, "Asymptotic Enumeration Methods," in Handbook of Combinatorics (R. Graham, M. Grötshel and L. Lovász ed), Elsevier Sci., vol.2, 1063-1230, 1995.

A.G. Pakes and F.M. Dekking, "On family trees and subtrees of simple branching processes," J. Theoret. Probab. vol. 4, 353-369, 1991.

R. Pemantle, "Phase transition in reinforced random walk and RWRE on trees," Ann. Probab. vol. 16, 1229-1241, 1988. 